\title{
CONSTRUINDO A POSSIBILIDADE DE PARTICIPAÇÃO DOS USUÁRIOS NAS CONFERÊNCIAS DE SAÚDE MUNICIPAIS: UM RELATO DE EXPERIÊNCIA
}

\section{BUILDING THE POSSIBILITY OF USER PARTICIPATION IN MUNICIPAL HEALTH CONFERENCES: AN EXPERIENCE REPORT}

\author{
Karen Silva de Castro ${ }^{1} *$ Lauany Silva de Medeiros $^{2} *$ Nayara Fernanda Alves Moreira ${ }^{3}$ Michele \\ Pinheiro Ferreira $^{4} *$ Amanda Ouriques de Gouveia ${ }^{5} *$ Aline Ouriques de Gouveia $^{6} *$ Laís Araújo $^{*}$ \\ Tavares Silva $^{7} *$ Carina Lopes de Sousa Antonio $^{8} *$ Valeria Regina Cavalcante dos Santos $^{9}$
}

\begin{abstract}
RESUMO
Objetivo: Analisar o processo de tomada de decisão durante a XII Conferência Municipal de Saúde do Conselho Municipal de Saúde, realizada no município de Tucuruí-PA. Metodologia: Trata-se de um estudo qualitativo do tipo descritivo-exploratório, que tem por base o método da problematização com aplicação do Arco de Maguerez para a fundamentação teórica e prática do estudo, baseando-se em cinco etapas, sendo a observação da realidade, pontos-chave, teorização, hipóteses de solução e a aplicação na realidade. Resultados: A gestão municipal e o Conselho Municipal de Saúde promoveram a XII Conferência Municipal de Saúde, em 13 de abril de 2019, contando com a presença de 80 pessoas, sendo cidadãos, prefeito, secretários, profissionais e acadêmico, entre a faixa etária de 18 a 60 anos de idade. Contudo, anteriormente, foram realizadas 04 reuniões do conselho, 02 pré-conferência, sendo uma na Unidade Básica de Saúde do Santa Mônica e a outra na Aldeia Indígena Teapykawa Assurini, por meio, de seminários, mesas redondas, assim como, debates abertos. Conclusão: Foi possível durante a conferência de saúde municipal abordar como os temas relativos ao princípio da participação da comunidade na construção de projetos de leis sobre a saúde são essenciais para a manutenção da universalidade, equidade e integralidade do Sistema Único de Saúde.
\end{abstract}

Palavras-Chave: Sistema Único de Saúde; Conferência de Saúde; Democracia; Participação Comunitária.

\begin{abstract}
Objective: To analyze the decision-making process during the 12th Municipal Health Conference of the Municipal Health Council, held in the municipality of Tucuruí-PA. Methodology: It is a qualitative study of the descriptive-exploratory type, which is based on the problematization method with the application of Arco de Maguerez for the theoretical and practical foundation of the study, based on five steps, being the observation of reality, key points, theorization, solution hypotheses and application in reality. Results: The municipal management and the Municipal Health Council promoted the 12th Municipal Health Conference, on April 13, 2019, with the presence of 80 people, being citizens, mayor, secretaries, professionals and academics, among the age group of 18 to 60 years old. However, previously, 04 council meetings were held, 02 pre-conference, one in the Basic Health Unit of Santa Monica and the other in the Teapykawa Assurini Indigenous Village, through seminars, round tables, as well as open debates. Conclusion: It was possible during the municipal health conference to address how themes related to the principle of community participation in the construction of draft health laws are essential for maintaining the universality, equity and integrality of the Unified Health System.
\end{abstract}

Keywords: Health Unic System; Health Conference; Democracy; Community Participation.

\footnotetext{
${ }^{1}$ Orcid: https://orcid.org/0000-0001-8463-0322

${ }^{2}$ Orcid: https://orcid.org/0000-0002-5683-6347

${ }^{3}$ Orcid: https://orcid.org/0000-0003-1094-7399

${ }^{4}$ Orcid: https://orcid.org/0000-0001-5316-9908

${ }^{5}$ Orcid: https://orcid.org/0000-0002-6874-8352

${ }^{6}$ Orcid: https://orcid.org/0000-0001-8743-1433

${ }^{7}$ Orcid: https://orcid.org/0000-0002-6517-3338

${ }^{8}$ Orcid: https://orcid.org/0000-0002-8980-4368

${ }^{9}$ Orcid: https://orcid.org/0000-0002-1264-8125
} 


\section{INTRODUÇÃO}

A Constituição Federal de 1988 promoveu a descentralização da gestão das políticas públicas, efetivando a participação da sociedade civil no processo de tomada de decisão sobre assuntos relacionados a saúde como direito geral. Dessa forma, normas e deliberações foram criadas como o intuito de proporcionar maior autonomia e efetiva participação da comunidade a respeito do processo de estruturação da atenção à saúde, sendo assim, a influência dos diversos elementos sociais nas instituições públicas foram seguramente ampliados e essenciais para o desenvolvimento dos serviços $\operatorname{prestados}^{(1)}$.

Outrossim, a formulação de regulamentações constitucionais estabelecidas pelo Sistema Único de Saúde (SUS), corroboraram para a efetivação de inovações institucionais como a formação de conferencias municipais, estaduais e nacionais e de conselhos gestores de políticas públicas, os quais tornaram-se essenciais para o fortalecimento do regime democrático na sociedade, possibilitando a ampliação dos seus direitos civis e contribuindo para o estreitamento das relações com os setores legislativos $^{(2)}$.

Ademais, segundo propõe a Lei $\mathrm{n}^{\circ}$ 8.142/1990, o Conselho de Saúde deve estabelecer estratégias capazes de promover a manutenção e controle das políticas de saúde, assim como estruturar os setores econômicos e financeiros, de modo que os serviços prestados sejam qualificados e capacitados cada vez mais, verificando as necessidades e carências e formulando maneiras de saná-las. Além disso, essa lei regulamenta o papel das Conferências de Saúde, elucidando suas conjunturas e finalidades, evidenciando que esse evento deve ocorrer a cada quatro anos, com o intuito de analisar e discutir as políticas públicas em cada esfera do governo e intentar diretrizes sobre o assunto a nível nacional, estadual e municipal, contribuindo para a verificação das demais políticas sociais, sendo elas correspondentes a política da mulher, criança e adolescente, assistência social, trabalhador, educação, idosos, meio ambiente e outras ${ }^{(3)}$.

Nessa perspectiva, os Conselhos e Conferências, são imprescindíveis para a manutenção de uma política pública de saúde integral, essencial para o fortalecimento e qualificação dos serviços ofertados, além de complementar a efetivação da participação da comunidade na formulação de deliberações, de forma que enfatize o valor da democracia frente a estruturação de medidas sociais de saúde, demonstrando que as discussões realizadas nesse ambiente propiciam a avaliação dos interesses e conflitos sociais, permeando ideias para a harmonização dos 
assuntos propostos e base para sanar as principais problemáticas impostas ${ }^{(4)}$.

Nesse interim, o estudo teve como objetivo analisar o processo de tomada de decisão durante a XII Conferência Municipal de Saúde do Conselho Municipal de Saúde, realizada no município de Tucuruí-PA, no ano de 2019, utilizando como base referencial, a influência das políticas públicas nas conferências municipais e como a representação popular pode atuar na formulação das deliberações para a qualificação e capacitação dos serviços de saúde prestados. Além disso, verificou-se a apresentação e importância desse elemento institucional para a melhora da premissa democrática e os setores legislativos.

\section{METODOLOGIA}

Trata-se de um estudo qualitativo do tipo descritivo-exploratório, que tem por base o método da problematização com aplicação do Arco de Maguerez para a fundamentação teórica e prática do estudo. O referido método, se baseia em cinco etapas, que são: A observação da realidade, pontos-chave, teorização, hipóteses de solução e a aplicação na realidade, sendo caracterizados como estímulos potenciais a processo educativo e $\operatorname{adaptativo}^{(5)}$.

Nessa perspectiva, a primeira fase, denominada de observação da realidade foi promovida por meio de debates feitos em reuniões do Conselho Municipal de Saúde, onde colocavam em pauta, questões das comunidades, reinvindicações e necessidades de cada bairro e também das comunidades indígenas, por meio de 3 reuniões em setores planejados. Em seguida, foi iniciada a segunda etapa que consistiu na definição das questões norteadoras, sendo: Quais as principais necessidades relacionadas as instituições de saúde? Como amenizar as problemáticas das comunidades? Quais as questões mais emergenciais? Como elencar e abranger todos os públicos?

A partir disso, seguiu-se para a formulação da terceira etapa, que consiste na teorização, promovida pela análise bibliográfica de dados buscados em sites e plataformas digitais como Scielo, LILACS e outras literaturas que servissem para o embasamento do estudo, as quais contemplavam conteúdos sobre Conferencias de Saúde, a importância delas e direitos dos cidadãos. Com isso, as bibliografias foram selecionadas e organizadas em um organograma seguindo os critérios de inclusão, como o recorte temporal de cinco anos e artigo completo, para então serem analisadas a participação popular durante uma conferência e o impacto desse processo na realidade social. 
Nesse sentido, ocorreu a quarta etapa para definir as hipóteses de solução, a fim de ordenar os planos que tinham como intuito integrar as demandas das comunidades e sanar os problemas existentes. Por fim, houve a quinta etapa para a aplicação na realidade que foi desenvolvida por meio de duas reuniões destinadas a população da cidade de TucuruíPA, onde foram levantas as informações, dúvidas e posicionamentos a respeito do Conselho de Saúde e seu papel na garantia dos direitos da comunidade e profissionais de saúde.

\section{RESULTADOS}

As Conferências de Saúde (CS) consistem em um avanço institucional embasado no princípio da democracia deliberada, no Brasil. A partir disso, caracterizam-se pelo apoio popular, na elaboração de políticas públicas destinadas a orientar os serviços de saúde atestados no Sistema Único de Saúde (SUS), desde 1986. Portanto, esse modelo de assistência ocorre, por meio, de uma via dupla, a primeira é a aderência, mobilização e representação social de diferentes comunidades, a fim de garantir o direito da equidade, principalmente, entre as parcelas marginalizadas e, em segundo, um representante político comprometido a dialogar com os envolvidos na CS, buscando criar um consenso entre os interesses de $\operatorname{todos}^{(6)}$.
Nesse sentido, o engajamento comunitário torna-se um pilar ao "controle social" do atendimento público, o qual representa uma conquista nacional para descentralizar e municipalizar as redes de atenção. Consequentemente, aconteceu uma transição em que usuário (paciente) assumiu a responsabilidade de avaliar a eficácia das medidas resolutivas em saúde, sendo assim, foi reconhecida a Lei $\mathrm{n}^{\circ} 8.142 / 90$ que dispõem a organização dos conselhos federal, estaduais e municipais de saúde, os quais afirmariam as bases do SUS, como universalização, integralidade, hierarquização e cooperação da sociedade ${ }^{(7)}$.

Desse modo, a gestão municipal de Tucuruí-Pa e o Conselho Municipal de Saúde promoveram a XII Conferência Municipal de Saúde, em 13 de abril de 2019, para ampliar fóruns de formação política e deliberação dos planos de ação destinados a promoção, proteção e recuperação da qualidade de vida dos indivíduos, bem como, definir sobre a alocação dos recursos previdenciários a essas decisões, com isso, contou com a presença de 80 pessoas, sendo cidadãos, prefeito, secretários, profissionais e acadêmico, entre a faixa etária de 18 a 60 anos de idade. Contudo, anteriormente, foram realizadas 04 reuniões do conselho, 02 pré-conferência, sendo uma na Unidade Básica de Saúde do Santa Mônica e a outra na Aldeia Indígena Teapykawa Assurini, por meio, de 
seminários, mesas redondas, assim como, debates abertos.

Nessa perspectiva, a conferência apresentava o tema gerador "Democracia e Saúde" que dividiu-se em 04 eixos de discussão, como I) Saúde como direito abordou sobre a criação do Conselho Nacional de Saúde, além da atual configuração paritária; II) Consolidação do SUS - tratou da Lei Orgânica n ${ }^{\circ} 8.080 / 90$ na formação do SUS; III) Financiamento do SUS - trabalhou o subfinanciamento do SUS associado a invenção do Prontuário Eletrônico do Cidadão (PEC) e IV) Democracia e Participação popular - conceituou a democracia, através, de uma dinâmica lúdica, na qual criaram 04 grupos, tendo o objetivo de elaborar propostas municipais e estatais para cada eixo, com isso, na plenária compareceram 39 representantes, dentre estas 05 ficaram na moção e 12 delegados foram escolhidas, entre os segmentos de "Gestão", "Trabalhador" e "Usuário" para serem encaminhados a Conferência Estadual de Saúde.

No que tange as limitações encontradas no evento, reporta-se 0 comparecimento de poucos moradores da cidade de Tucuruí, localizado na região sudeste do estado do Pará, associada à uma divulgação ineficiente, tendo em vista que o município apresenta um vasta extensão geoespacial e a necessidade de que uma maioria estivesse presente neste evento, pois é, dessa maneira, que os lideres estarão enterrados das reais condições dos serviços de saúde, da eficiência dos cuidados prestados, dos programas que estão ativos e das pendenciais da unidade de cada bairro.

Diante disso, como medida de solução propõem-se articular estratégias de marketing pelas quais serão formadas redes de contato com as comunidades, como páginas sociais do conselho de saúde, na qual divulgariam decisões relevantes a conferência, assim como, posts sobre o compromisso social em atuar nessas ocasiões, além disso, a utilizações de ferramentas públicas para fazer os convites, informando data local e hora, em geral, se utilizaria o carro-som e, a nível local, as UBS para distribuição de panfletos.

\section{DISCUSSÃO}

\section{Perspectiva dos encontros no conselho de} saúde:

Os conselhos de saúde representam um órgão de controle social, pois o setor coletivo e governamental precisa, de forma integrada, articular e implementar os serviços, programas, protocolos de saúde, com o fundamento de vigilância e compromisso integral, por isso a composição dos membros deve ser entre usuários e representantes governamentais. No município de Tucuruí-PA 
a coordenação do Conselho de Saúde é formada por um presidente, primeiro secretário, secretário executivo, secretário de saúde e conselheiro elegidos democraticamente, segundo os princípio ético-político-ideológico ${ }^{(8)}$.

Em vista disso, o nível municipal possui o compromisso de mobilizar os grupos sociais para agregarem a causa social de realizar as conferências, tanto locais quanto distritais, a fim de transforma-los nos verdadeiros protagonistas do "fazer saúde", por meio, do reconhecimento de direitos e deveres como usuários do $\mathrm{SUS}^{(9)}$. Logo, as reuniões do conselho de Tucuruí ocorreram entre os meses de março e abril de 2019, sendo ao total 04 encontros, no horário de $17 \mathrm{~h} 00 \mathrm{~min}$ às $18 \mathrm{~h} 30 \mathrm{~min}$, dos conselheiros, professores e acadêmicos de enfermagem das instituições de ensino Universidade Estadual do Pará (UEPA) e Faculdade Gamaliel.

A partir disso, as conversas eram salvas, por um aparelho gravador, para arquivar as pautas levantadas, bem como, as decisões alcanças ao fim da assembleia. Com isso, os principais pontos abordados foram às datas das pré-conferências, sendo firmado que a da UBS Santa Mônica fosse em 03 de abril de 2019, com início às 18h00min (período vespertino), e a da aldeia Teapykawa Assurini em 21 de abril de 2019, às 09h00min (período matutino), a data e localidade da conferência municipal, as quais consistiram ao dia 12 e 13 de abril de 2019, no estabelecimento Sabor dos Sonhos Eventos, respectivamente.

Logo, houve a proposta de dividir os acadêmicos em comissões organizativas, com a intenção de otimizar as tarefas da conferência, com isso formou-se 3 equipes para "Relatoria", os quais escreveriam a ata com as propostas escolhidas na plenária e a descrição das atividades desenvolvidas em cada nos dois dias; "Organização" que ficaram encarregados da arrumação do local, como delegação dos espaços para a mesa redonda, credenciamento, limite de tempo das plenárias e as palestrantes de cada eixo; "Credenciamento" responsáveis por recolher a assinatura de todos os participantes, visando a entrega de certificado do evento com carga horária de 40 horas.

\section{CONSIDERAÇÕES FINAIS}

Logo, foi possível durante a conferência de saúde municipal abordar como os temas relativos ao princípio da participação da comunidade na construção de projetos de leis sobre a saúde são essenciais para a manutenção da universalidade, equidade e integralidade do Sistema Único de Saúde. Nesse sentido, o controle social emerge como efeito dessa participação, que tem por objeto não estritamente $\mathrm{o}$ setor saúde, mas a compreensão desse campo em suas 
implicações recíprocas com as políticas sociais e econômicas.

\section{REFERÊNCIAS}

1. Kruger T, Lemke AP, Nardino D, Finger SJ, Meggiato JR, Nunes MLA, Pedrini DM. The propositions of Health Conferences and Municipal Health Plans: a study in municipalities of Santa Catarina. Health in Debate [Internet]. December 2011[cited 2020 june 8]; 35(91): 508-521. Available from: https://www.redalyc.org/pdf/4063/406341 765003.pdf.

2. Müller JS, Artmann E. Discourses on the role and representativeness of municipal health conferences. Sergio Arouca National School of Public Health, Oswaldo Cruz Foundation [Internet]. January 2014 [cited 2020 april 20]; 30 (1): 68-78. Available from: https://www.scielosp.org/article/csp/2014. v30n1/68-78/pt/.

3. Souza AVD, Krüger TR. Social participation in the SUS: proposals from conferences on the local health council. Journal of Public Health of Santa Catarina [Internet]. 2010 [cited 2020 april 04]; 3(1), 80-96. Available from: https://d1wqtxts1xzle7.cloudfront.net/363 34992/72-275-1-PB-with-cover-pagev2.pdf.

4. Faria CF, Lins IL, Lobão ER, Cardoso JAP, Petinelli V. Local, district and municipal health conferences: change of scale and formation of a participatory, representative and deliberative public policy system [Internet]. 2012[cited 2020 june 05]; Text for Discussion. Available from:

https://www.ipea.gov.br/portal/index.php? option=com_content $\&$ view $=$ article \&id $=1$ 509.
5. Berbel NN. "Problematization" and Problem-Based Learning: different words or different ways? Interface Communication, Health, Education [Internet]. 1998 [cited 2020 june may 22; 2(2)1,139-154. Available from: https://www.scielo.br/j/icse/a/BBqnRMcd xXyvNSY3YfztH9J/?format=pdf\&lang $=p$ t.

6. Neto JSM, Artmann E. Discourses on the role and representativeness of municipal health conferences. Caderno de Saúde Pública. [Internet]. 2014 [cited $2020 \mathrm{Jul}$ 15]; 30(01): 68-78. Available from: https://europepmc.org/article/med/246270 14.

7. Tofani LFN, Carpenter MDCC. 3rd Municipal Health Conference of Várzea Paulista: society's participation in the process of prioritization and political commitment. Saúde e Sociedade. [Internet].2012 [cited 2020 Jul 02]; 21(01): 244-252. Available from: https://www.scielo.br/j/sausoc/a/D9FmgX sR9ZLW66J5HH8zqKq/?lang=pt.

8. Rolim LB, Cruz RSBLC, Sampaio KJAJ. Popular participation and social control as a SUS guideline: a narrative review. Revista Saúde em Debate. [Internet]. 2013 [cited 2020 Jul 05]; 37(96): 139-147. Available from: https://www.scielo.br/j/sdeb/a/dNgCW9W dJJx7VHV7 $\times$ WkhSHq/?lang=pt\&format $=$ pdf.

9. Wendhausen A, Caponi S. Dialogue and participation in a health council in Santa Catarina, Brazil. Caderno de Saúde Pública. [Internet]. 2002 [cited 2020 Jul 12]; 18(06):01-03. Available from: https://digitalrepository.unm.edu/lasm_en/ $239 /$.

Submissão: 2021-02-18

Aprovado: 2021-06-22 